\title{
Reproducción de Eisenia foetida en suelos agrícolas de áreas mineras contaminadas por cobre y arsénico
}

\author{
Gonzalo Ávila(1), Hernán Gaete $^{(1)}{\text { Mario } \text { Morales }^{(1)} \text { y Alexander Neaman }}^{(2)}$
}

\begin{abstract}
(1)Universidad de Valparaíso, Facultad de Ciencias, Departamento de Biología y Ciencias Ambientales, Av. Gran Bretaña 1.111, Playa Ancha, Valparaíso, Chile. E-mail: gonzaloaa2000@yahoo.com, hernan.gaete@uv.cl, mzmorales@gmail.com (2)Universidad Católica de Valparaíso, Facultad de Agronomía, Área Medio Ambiente, San Francisco s/n, La Palma, Casilla 4-D, Quillota, Chile. E-mail: alexander.neaman@ucv.cl
\end{abstract}

\begin{abstract}
Resumen - El objetivo del trabajo fue evaluar la toxicidad del cobre y arsénico en suelos agrícolas, mediante bioensayos estandarizados de toxicidad aguda y crónica sobre Eisenia foetida y relacionar la respuesta de ésta con las concentraciones de cobre y arsénico en los suelos. Los suelos agrícolas fueram muestreados en las inmediaciones de áreas mineras en la cuenca del río Aconcagua, Chile. E. foetida expuesta a los suelos estudiados mostró una baja mortalidad, indicando la ausencia de toxicidad aguda. Además, se observó una disminución en la producción de capullos y desarrollo de juveniles, indicando la existencia de toxicidad crónica. Dicha disminución fue relacionada con las concentraciones de cobre y arsénico en los suelos. El cobre y arsénico en conjunto disminuyeron la producción de capullos, mientras que sólo el arsénico disminuyó el desarrollo de juveniles. Suelos agrícolas provenientes de áreas mineras de la cuenca poseen potenciales características tóxicas para el desarrollo de la macrofauna edáfica.
\end{abstract}

Términos para indexación: lombriz de tierra, bioensayo, toxicidad.

\section{Reproduction of Eisenia foetida in agricultural soils from mining areas contaminated with copper and arsenic}

\begin{abstract}
The objective of this work was to evaluate copper and arsenic toxicity in agricultural soils, by means of standardized bioassays of chronic and acute toxicity on Eisenia foetida, and to relate E. foetida response to copper and arsenic concentrations in soils. The agricultural soils were sampled in the proximity of copper mining industries, in the Aconcagua river basin, Chile. E. foetida exposed to the studied soils exhibited a low mortality, indicating the absence of acute toxicity. Besides, a decrease in the cocoon production and juvenile development was observed, indicating the existence of chronic toxicity. Reduction in the cocoon production and juvenile development was related to soil concentrations of copper and arsenic. Both copper and arsenic reduced the cocoon production, while only arsenic diminished the juvenile development. Agricultural soils from mining areas of the basin have potentially toxic characteristics for the development of soil macrofauna.
\end{abstract}

Index terms: earthworm, bioassay, toxicity.

\section{Introducción}

Las lombrices de tierra, junto con otros organismos macrodescomponedores, están entre los organismos más importantes del suelo. Esto se debe a la capacidad de descomponer la materia orgánica, reciclamiento de nutrientes y formación de suelo (Raty \& Huhta, 2004). Esta importante función puede ser afectada por la presencia de elementos químicos tóxicos en el suelo (Spurgeon et al., 1994).

El cobre y el arsénico son importantes contaminantes ambientales asociados principalmente a las actividades mineras (De Gregori et al., 2003). Estos alteran las características naturales de los suelos en sectores cercanos a dichas actividades y pueden provocar efectos adversos sobre los organismos vivos del suelo. El cobre y el arsénico pueden ser tóxicos para todos los organismos dependiendo de las concentraciones en que se encuentren (Adriano, 2001).

Una aproximación en la evaluación de los efectos de los agentes tóxicos en suelos se realiza mediante bioensayos de toxicidad. Para ello se han desarrollado metodologías estandarizadas (Organisation for Economic Cooperation and Development, 1984, 2000), las cuales se llevan a cabo para determinar los efectos agudos y crónicos en lombrices de tierra. Estas pruebas evalúan 
la contaminación del suelo, a través de indicadores como mortalidad, reproducción u otra respuesta subletal en los organismos de prueba.

Se ha encontrado que las lombrices de tierra son menos sensibles a suelos recolectados en terreno que a suelos artificialmente contaminados. Esto se debe a una mayor biodisponibilidad de elementos en los últimos (Spurgeon \& Hopkin, 1995, 1996). Es por esto que el uso de suelos recolectados en terreno refleja, de mejor manera, la interacción entre los elementos del suelo con los organismos asociados a éste en condiciones naturales.

En Chile, los estudios que relacionan elementos traza con organismos asociados al suelo están referidos sólo a plantas que son cultivadas en suelos agrícolas. En estos estudios se ha analizado la transferencia de cobre a tejidos, en diversas especies de hortalizas (De Gregori et al., 2000; Badilla-Ohlbaum et al., 2001; Ginocchio et al., 2002). Es importante destacar la creciente utilización de bioensayos de ecotoxicidad con lombrices de tierra, especialmente en Europa (Spurgeon et al., 2003). Sin embargo, en Chile no existen estudios donde se utilicen estas metodologías biológicas.

En esta investigación, se propuso determinar la toxicidad del cobre y arsénico, en suelos agrícolas de la cuenca del río Aconcagua, mediante bioensayos estandarizados de toxicidad aguda y crónica sobre E. foetida, y relacionar la respuesta de ésta con las concentraciones de cobre y arsénico en los suelos.

\section{Material y Métodos}

Se tomaron diez muestras de suelos agrícolas, en diferentes localidades en la cuenca del río Aconcagua y se determinaron sus coordenadas geográficas: Los Andes

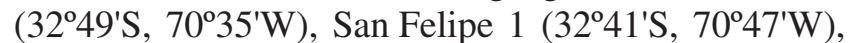
San Felipe $2\left(32^{\circ} 41^{\prime} \mathrm{S}, 70^{\circ} 45^{\prime} \mathrm{W}\right)$, San Felipe $3\left(32^{\circ} 44^{\prime} \mathrm{S}\right.$, $\left.70^{\circ} 46^{\prime} \mathrm{W}\right)$, Chagres $1\left(32^{\circ} 48^{\prime} \mathrm{S}, 70^{\circ} 57^{\prime} \mathrm{W}\right)$, Chagres 2 $\left(32^{\circ} 48^{\prime} \mathrm{S}, 70^{\circ} 57^{\prime} \mathrm{W}\right)$, Chagres $3\left(32^{\circ} 46^{\prime} \mathrm{S}, 70^{\circ} 59^{\prime} \mathrm{W}\right)$, Catemu $1\left(32^{\circ} 46^{\prime} \mathrm{S}, 70^{\circ} 58^{\prime} \mathrm{W}\right)$, Catemu $2\left(32^{\circ} 46^{\prime} \mathrm{S}\right.$, $\left.70^{\circ} 58^{\prime} \mathrm{W}\right)$ y El Melon $\left(32^{\circ} 39^{\prime} \mathrm{S}, 71^{\circ} 17^{\prime} \mathrm{W}\right)$. Los puntos de muestreo se establecieron en áreas cercanas a actividades mineras (fundición y tranques de relave). Las muestras de suelo $(10 \mathrm{~kg})$ fueron tomadas entre $0 \mathrm{y}$ $20 \mathrm{~cm}$ de profundidad, removiendo la vegetación existente. Estas fueron procesadas según Spurgeon \& Hopkin (1996).

Se determinó la textura del suelo según Van der Watt (1966). La materia orgánica fue determinada según Sadzawka et al. (2006). El cobre total fue determinado por espectroscopia de absorción atómica, después de la digestión ácida de las muestras con una mezcla de ácidos fluorhídrico y perclórico (Maxwell, 1968). La determinación de la concentración de arsénico total se realizó mediante análisis por activación neutrónica (International Atomic Energy Agency, 2001).

Para determinar las formas en que se encuentran el cobre y el arsénico en los suelos estudiados, se realizaron análisis por medio de microsonda electrónica (Amonette, 2002). Los suelos fueron impregnados con una resina epóxica bajo vacío. Posteriormente, secciones finas fueron preparadas y pulidas (National Petrographic Service, 2007).

Para la determinación de $\mathrm{pH}$, cobre soluble, cobre libre $\left(\mathrm{Cu}^{2+}\right)$ y lábil se usó una solución de $\mathrm{KNO}_{3} 0,1 \mathrm{M}$ como extractor. Las concentraciones de cobre y arsénico solubles, al inicio y término del bioensayo, fueron determinadas por espectroscopia de absorción atómica y espectroscopia de fluorescencia atómica acoplada a generación de hidruros, respectivamente (De Gregori et al., 2003).

El cobre libre $\left(\mathrm{Cu}^{2+}\right)$ se determinó con el uso de un electrodo de ión selectivo (Sauvé et al., 1995). El cobre lábil fue determinado por voltametría (Martínez \& McBride, 1998, 1999). La fracción lábil incluye al ión libre $\mathrm{Cu}^{2+}$ y complejos lábiles de cobre que pueden disociarse rápidamente y producir $\mathrm{Cu}^{2+}$. Los complejos lábiles incluyen complejos inorgánicos y complejos orgánicos débiles (por ejemplo con acetato, formato y lactato). Por otra parte, complejos orgánicos fuertes (con substancias húmicas) no son lábiles (Martínez \& McBride, 1998, 1999).

Para determinar la toxicidad aguda y crónica, se llevaron a cabo bioensayos con $E$. foetida según métodos estandarizados por la Organisation for Economic Cooperation and Development (1984, 2000). Para ello, $500 \mathrm{~g}$ de suelo fueron ajustados a una humedad del $40 \%$, en cada contenedor experimental de vidrio de $750 \mathrm{~mL}$. En el bioensayo de toxicidad aguda, se incubaron treinta lombrices jóvenes de la especie E. foetida (sin desarrollo de clitelium), distribuidas en tres réplicas. Los organismos no fueron alimentados durante el experimento. La sobrevivencia de las lombrices fue medida al término de dos semanas de exposición.

Para determinar la toxicidad crónica, se incubaron diez lombrices adultas de E. foetida (con clitelium visible), anteriormente lavadas con agua destilada, secadas en papel filtro y pesadas individualmente. Como alimento se aplicó $5 \mathrm{~g}$ de estiércol de vaca humedecido con $5 \mathrm{~mL}$ de agua destilada. Para acostumbrar las 
lombrices, se las alimentó con dicho alimento una semana antes de la realización del bioensayo. Se realizaron ocho replicas por cada muestra y el tiempo de exposición fue ocho semanas. Al término de las primeras cuatro semanas, se determinaron los promedios de peso, el número de lombrices sobrevivientes y el número de capullos producidos. Al finalizar las ocho semanas, se determinó la cantidad promedio de juveniles desarrollados. Durante el periodo de los bioensayos, la temperatura ambiental fue mantenida en un rango de $22-24^{\circ} \mathrm{C}$, con una iluminación de 400 lux y un fotoperíodo de 12 horas de luz y 12 horas de oscuridad. La humedad fue mantenida por medio de la aplicación de $40 \mathrm{~mL}$ de agua destilada una vez a la semana.

Para comparar la respuesta de E. foetida, obtenida en los bioensayos entre estaciones de muestreo, se realizó un test de comparaciones múltiples de Tukey, al $5 \%$ de probabilidad. Se realizaron correlaciones de Pearson entre las respuestas biológicas y las concentraciones de cobre y arsénico.

\section{Resultados y Discusión}

Los suelos estudiados presentaron, en general, una textura franca (Cuadro 1). Las concentraciones de materia orgánica variaron entre 20 y $60 \mathrm{~g} \mathrm{~kg}^{-1}$, y los valores de $\mathrm{pH}$ fueron neutros. Los suelos fueron clasificados como Entisoles (Soil Survey Staff, 1999).

Según el análisis cualitativo, realizado por medio de microsonda electrónica, el cobre en los suelos estudiados se encontraba principalmente como sulfuros. Además, el cobre se encontraba en óxidos de hierro en concentraciones $290-3.250 \mathrm{mg} \mathrm{kg}^{-1}$ (promedio $\left.1.440 \mathrm{mg} \mathrm{kg}^{-1}\right)$. El cobre puede sustituir el hierro en la estructura de los óxidos de hierro (Cornell \& Schwertmann, 1996) o puede estar adsorbido sobre dichos óxidos (McBride, 1994).
El arsénico se encontraba principalmente en óxidos de hierro, en concentraciones $240-1310 \mathrm{mg} \mathrm{kg}^{-1}$ (promedio $570 \mathrm{mg} \mathrm{kg}^{-1}$ ). El arsénico puede estar adsorbido sobre dichos óxidos (McBride, 1994; Adriano, 2001). Además, el arsénico se encontraba en los sulfuros de cobre en concentraciones $210-290 \mathrm{mg} \mathrm{kg}^{-1}$ (promedio $240 \mathrm{mg} \mathrm{kg}^{-1}$ ). El arsénico está, generalmente, presente en los sulfuros de cobre (O’Neill, 1995).

El cobre soluble representó el $0,1 \%$ del cobre total (Cuadro 2). Las concentraciones de cobre soluble, medidas al término de los bioensayos, bajaron con respecto a las iniciales. Eso se podría deber a una complejación de cobre por la materia orgánica presente en la fase sólida (McBride, 1994). Las concentraciones de cobre libre $\left(\mathrm{Cu}^{2+}\right)$ fueron inferiores al 0,5\% del cobre en la solución, lo que sugiere que prácticamente todo el cobre soluble estaba presente como complejos orgánicos (Sauvé et al., 1997). El cobre libre $\left(\mathrm{Cu}^{2+}\right)$ aumentó al término de los bioensayos. Eso se podría deber a una menor presencia de ácidos orgánicos en la solución (McBride et al., 1997; Sauvé et al., 1997).

El arsénico soluble representó valores menores que 0,001\% del arsénico total. Las concentraciones de arsénico soluble bajaron al término de los bioensayos. Los mecanismos que controlan la solubilidad de arsénico en los suelos son complejos (Adriano, 2001) y, en este estudio, no se pudo suponer a que se debe este cambio en la solubilidad de arsénico al término de los bioensayos.

En los bioensayos de toxicidad, las lombrices expuestas por un periodo de dos semanas presentaron una sobrevivencia de $100 \%$ en todas las estaciones de muestreo estudiadas, indicando una ausencia de toxicidad aguda. Las lombrices expuestas por un periodo de cuatro semanas presentaron una sobrevivencia mayor que $90 \%$ en todas las estaciones de muestreo (Cuadro 3). Spurgeon \& Hopkin (1995) encontraron toxicidad aguda en lombrices de tierra $E$. foetida expuestas a suelos

Cuadro 1. Características químicas y textura de las muestras de suelos agrícolas en la cuenca del río Aconcagua.

\begin{tabular}{|c|c|c|c|c|c|c|}
\hline Estación $^{(1)}$ & $\mathrm{pH}$ & $\mathrm{MO}^{(2)}\left(\mathrm{g} \mathrm{kg}^{-1}\right)$ & Arena $\left(\mathrm{g} \mathrm{kg}^{-1}\right)$ & $\operatorname{Limo}\left(\mathrm{g} \mathrm{kg}^{-1}\right)$ & Arcilla $\left(\mathrm{g} \mathrm{kg}^{-1}\right)$ & Textura \\
\hline LA & 6,3 & 46 & 450 & 350 & 200 & Franca \\
\hline SF1 & 6,9 & 41 & 350 & 400 & 250 & Franca \\
\hline SF2 & 6,7 & 19 & 575 & 300 & 125 & Franca arenosa \\
\hline SF3 & 6,4 & 50 & 450 & 400 & 150 & Franca \\
\hline $\mathrm{CH} 1$ & 7,1 & 19 & 400 & 525 & 75 & Franco limosa \\
\hline $\mathrm{CH} 2$ & 6,9 & 29 & 700 & 275 & 25 & Franco arenosa \\
\hline $\mathrm{CH} 3$ & 7,1 & 32 & 700 & 250 & 50 & Franco arenosa \\
\hline $\mathrm{C} 1$ & 6,5 & 62 & 850 & 125 & 25 & Areno franca \\
\hline $\mathrm{C} 2$ & 6,4 & 31 & 525 & 400 & 75 & Franca \\
\hline M & 6,7 & 47 & 625 & 275 & 100 & Franco arenosa \\
\hline
\end{tabular}

(1)LA: Los Andes; SF: San Felipe; CH: Chagres; C: Catemu; M: El Melón. ${ }^{(2)}$ Materia orgánica. 
recolectados en terreno con una concentración de $2.600 \mathrm{mg} \mathrm{kg}^{-1}$ de cobre total. En el presente estudio, las máximas concentraciones de cobre total fueron inferiores a dicho valor. Scott-Fordsmand et al. (2000) no evidenciaron cambios en la sobrevivencia en lombrices de tierra, expuestas a concentraciones hasta $1.400 \mathrm{mg} \mathrm{kg}^{-1}$ de cobre total en suelos recolectados en terreno. Lo anterior está en concordancia con los resultados encontrados en el presente estudio, donde no se observó efecto significativo en la sobrevivencia a concentraciones menores que $1.570 \mathrm{mg} \mathrm{kg}^{-1}$, en $2 \mathrm{y}$ 4 semanas.

En cuanto al arsénico presente en los suelos estudiados, no se observó mortalidad a la concentración más alta encontrada que fue $35 \mathrm{mg} \mathrm{kg}^{-1}$. Estos resultados son diferentes de lo reportado por Fischer \& Koszorus (1992), quienes utilizaron suelos orgánicos preparados en laboratorio y encontraron mortalidad cercana al $10 \%$ a $50 \mathrm{mg} \mathrm{kg}^{-1}$ de arseniato de potasio (equivalente a $29 \mathrm{mg} \mathrm{kg}^{-1}$ de arsénico total) para E. foetida. Esta diferencia se podría deber a diferencias en las metodologías usadas. Específicamente, la mortalidad fue determinada a las 8 semanas de exposición en el trabajo de Fischer \& Koszorus (1992), en comparación a la determinación a las 2 y 4 semanas en el presente estudio. No obstante, dicha diferencia también se podría deber a las diferencias de disponibilidad química del arsénico en los suelos artificiales y los recolectados en terreno. Fischer \& Koszorus (1992) agregaron arsénico como arseniato de potasio, el cual posee una alta biodisponibilidad y toxicidad. Esto se debe a que la materia orgánica del suelo posee carga negativa (Sparks, 2003), la cual no disminuye la disponibilidad de aniones (como el arseniato). En contraste, en los suelos recolectados en terreno, la biodisponibilidad y biotoxicidad están limitadas por la solubilidad de los compuestos. En el presente estudio, el arsénico de las muestras se encontraba en los sulfuros de cobre y óxidos de hierro, las formas que tienen baja solubilidad. Lo anterior resalta la importancia de utilizar suelos recolectados en terreno para la evaluación de las concentraciones letales de los contaminantes en el suelo.

El cambio de peso de las lombrices de tierra mostró la tendencia de disminuir durante los bioensayos

Cuadro 2. Cobre y arsénico de las muestras de suelos agrícolas en la cuenca del río Aconcagua(1).

\begin{tabular}{|c|c|c|c|c|c|c|c|c|c|}
\hline Estación & $\mathrm{Cu}$ total $^{(2)}$ & $\mathrm{Cu}$ soluble ${ }^{(2)}$ & Cu soluble ${ }^{(3)}$ & $\mathrm{Cu}^{2+(2)}$ & $\mathrm{Cu}^{2+(3)}$ & Cu lábil ${ }^{(2)}$ & As total ${ }^{(2)}$ & As soluble ${ }^{(2)}$ & As soluble ${ }^{(3)}$ \\
\hline & $\left(\mathrm{mg} \mathrm{kg}^{-1}\right)$ & |---------------- & ----------------- & $\left(\mu \mathrm{g} \mathrm{kg}^{-1}\right.$ & ------- & ----------- & $\left(\mathrm{mg} \mathrm{kg}^{-1}\right)$ & \multicolumn{2}{|c|}{----------( $\left.\mu \mathrm{g} \mathrm{kg}^{-1}\right)$---------- } \\
\hline LA & 117 & 88 & $-(4)$ & 0,22 & 0,35 & 2,6 & 34 & 2,3 & 2,1 \\
\hline SF1 & 822 & 430 & 158 & 0,27 & 0,34 & 12 & 24 & 9,0 & 4,3 \\
\hline SF2 & 65 & $-(4)$ & $-(4)$ & 0,06 & 0,11 & $-(4)$ & 28 & 8,0 & 7,0 \\
\hline SF3 & 533 & 505 & 386 & 1,60 & 2,74 & 66 & 32 & 4,9 & 2,4 \\
\hline $\mathrm{CH} 1$ & 274 & 203 & 277 & 0,06 & 0,28 & 0,4 & 35 & 20 & 21 \\
\hline $\mathrm{CH} 2$ & 1.570 & 1.340 & 1.040 & 0,63 & 2,19 & 105 & 30 & 26 & 22 \\
\hline $\mathrm{CH} 3$ & 261 & 128 & 87 & 0,005 & 0,01 & -(4) $^{(4)}$ & 26 & 19 & 27 \\
\hline $\mathrm{C} 1$ & 782 & 1.188 & 909 & 0,55 & 1,61 & 27 & 31 & 18 & 6,5 \\
\hline $\mathrm{C} 2$ & 180 & - 4 ) & -(4) & 0,10 & 0,18 & -(4) & 14 & 2,8 & 2,0 \\
\hline M & 82 & -(4) $^{(4)}$ & $-^{(4)}$ & 0,03 & 0,22 & $-^{(4)}$ & 18 & 4,0 & 1,0 \\
\hline
\end{tabular}

${ }^{(1)}$ LA: Los Andes; SF: San Felipe; CH: Chagres; C: Catemu; M: El Melón. ${ }^{(2)}$ Medición al inicio del bioensayo. (3)Medición al término del bioensayo.

${ }^{(4)}$ Bajo límite de detección para cobre soluble $\left(<75 \mu \mathrm{g} \mathrm{kg}^{-1}\right)$ y bajo límite de detección para cobre lábil $\left(<1,6 \mu \mathrm{g} \mathrm{kg}^{-1}\right)$.

Cuadro 3. Sobrevivencia (\%), cambio de peso (\%), producción de capullos y desarrollo de juveniles, en los bioensayos de toxicidad crónica sobre Eisenia foetida (Savigny) de suelos agrícolas en la cuenca del río Aconcagua ${ }^{(1)}$.

\begin{tabular}{|c|c|c|c|c|}
\hline Estación & Sobrevivencia $^{(2)}$ & Cambio de peso $^{(2)}$ & Producción de capullos ${ }^{(2)}$ & Desarrollo de juveniles $^{(3)}$ \\
\hline$\overline{\mathrm{LA}}$ & 100 & -1 & 3 & 10 \\
\hline SF1 & 100 & 5 & 3 & 14 \\
\hline SF2 & 100 & -13 & 2 & 11 \\
\hline SF3 & 93 & -15 & 1 & 11 \\
\hline $\mathrm{CH} 1$ & 100 & -12 & 1 & $2 *$ \\
\hline $\mathrm{CH} 2$ & 95 & -29 & 0 & $1 *$ \\
\hline $\mathrm{CH} 3$ & 98 & -8 & 3 & 6 \\
\hline $\mathrm{C} 1$ & 100 & -12 & 2 & 7 \\
\hline $\mathrm{C} 2$ & 100 & 7 & 4 & 12 \\
\hline $\mathrm{M}$ & 100 & -22 & $5^{*}$ & 8 \\
\hline
\end{tabular}

${ }^{(1)}$ LA: Los Andes; SF: San Felipe; CH: Chagres; C: Catemu; M: El Melón. ${ }^{(2)}$ Después de cuatro semanas. ${ }^{(3)}$ Después de ocho semanas. *Significativamente diferente de las otras muestras por Tukey test con una probabilidad de $5 \%$. 
(Cuadro 3). Se observó una relación entre la máxima perdida de peso y la máxima concentración de cobre total y soluble en la estación $\mathrm{CH} 2$. Sin embargo, los cambios de peso no se correlacionaron significativamente con las concentraciones de cobre y arsénico (Cuadro 4). Por lo tanto, cambio de peso no es un buen indicador de toxicidad.

Se observó una variación en la producción de capullos, entre 0,4 capullo en la estación $\mathrm{CH} 2$ y 4,5 capullos en la estación $\mathrm{M}$, en promedio. Esta última estación se diferenció significativamente de las otras muestras (Cuadro 3).

No se observó una correlación significativa entre la producción de capullos y las concentraciones de cobre total. Sin embargo, se encontró una correlación entre la producción de capullos y las concentraciones de cobre soluble, libre y lábil. Esta misma correlación se observó con el arsénico total (Cuadro 4). Las menores producciones de capullos fueron en las estaciones SF3, $\mathrm{CH} 1$ y CH2 (Figura 1) a concentraciones mayores que $260 \mu \mathrm{g} \mathrm{kg}^{-1}$ de cobre soluble, destacando la estación CH2 con una nula producción. En cuanto al arsénico total, la mayor producción de capullos fue en las estaciones con la menor concentración de arsénico (C2 y M).

Scott-Fordsmand et al. (2000) utilizaron suelos recolectados en terreno y determinaron una reducción significativa en la producción de capullos a $1.400 \mathrm{mg} \mathrm{kg}^{-1}$ de cobre total, resultado similar al encontrado en la estación $\mathrm{CH} 2$ con $1.570 \mathrm{mg} \mathrm{kg}^{-1}$. Por otro lado, Fischer \& Koszorus (1992) utilizaron suelos artificiales y observaron una reducción cercana al $30 \%$ en la producción de capullos, en relación al control, a una concentración de $50 \mathrm{mg} \mathrm{kg}^{-1}$ de arseniato de potasio (equivalente a $29 \mathrm{mg} \mathrm{kg}^{-1}$ de arsénico total). En el presente estudio, no se observó una reducción significativa a esas concentraciones. Esto sugiere que los suelos recolectados en terreno, en comparación con los suelos contaminados en laboratorio, reflejan de mejor manera la interacción entre los elementos del suelo con sus organismos asociados.

Para determinar efectos tóxicos en lombrices de tierra, generalmente se determinan las concentraciones individuales de los agentes contaminantes presentes en el suelo (Organisation for Economic Cooperation and Development, 1984, 2000). Sin embargo, esto no permite predecir la toxicidad de diferentes agentes químicos en conjunto. Los análisis de correlaciones múltiples encontraron una significativa correlación entre la producción de capullos y las concentraciones de cobre y arsénico totales (coeficiente de correlación múltiple, $\mathrm{r}=0,80 ; \mathrm{P}=0,05)$. Esto sugiere que cobre y arsénico totales, en conjunto, explican una proporción mayor de la variabilidad de la producción de capullos, que por separado.

Se ha reconocido que la determinación de las formas biodisponibles de los elementos traza en los suelos es más útil para evaluar la toxicidad en los organismos, en comparación con la determinación de concentraciones totales de dichos elementos (Lanno et al., 2003; Janssen et al., 2005). Sin embargo, con los resultados obtenidos en esta investigación, no queda claro qué forma, total o biodisponible de los elementos, es la más adecuada para determinar efectos sobre las respuestas de los bioensayos de toxicidad.

Cuadro 4. Correlaciones de Pearson entre las concentraciones de diferentes formas de Cu o As y las respuestas biológicas.

\begin{tabular}{|c|c|c|c|c|c|}
\hline Forma del elemento & Parámetro biológico & Coeficiente r (inicio) & $\mathrm{P}$ & Coeficiente r (término) & $\mathrm{P}$ \\
\hline \multirow[t]{3}{*}{$\mathrm{Cu}$ total } & Cambio de peso & $-0,389$ & 0,267 & & \\
\hline & Producción de capullos & $-0,622$ & 0,055 & & \\
\hline & Desarrollo de juveniles & $-0,394$ & 0,260 & & \\
\hline \multirow[t]{3}{*}{$\mathrm{Cu}$ soluble } & Cambio de peso & $-0,473$ & 0,167 & $-0,547$ & 0,102 \\
\hline & Producción de capullos & $-0,628$ & 0,052 & $-0,694$ & $0,026^{*}$ \\
\hline & Desarrollo de juveniles & $-0,444$ & 0,198 & $-0,571$ & 0,084 \\
\hline \multirow[t]{3}{*}{$\mathrm{Cu}^{2+}$} & Cambio de peso & $-0,279$ & 0,435 & $-0,504$ & 0,138 \\
\hline & Producción de capullos & $-0,533$ & 0,112 & $-0,663$ & $0,037 *$ \\
\hline & Desarrollo de juveniles & 0,077 & 0,833 & $-0,218$ & 0,545 \\
\hline \multirow[t]{3}{*}{ Cu lábil } & Cambio de peso & $-0,593$ & 0,071 & & \\
\hline & Producción de capullos & $-0,710$ & $0,022 *$ & & \\
\hline & Desarrollo de juveniles & $-0,381$ & 0,277 & & \\
\hline \multirow[t]{3}{*}{ As total } & Cambio de peso & $-0,293$ & 0,412 & & \\
\hline & Producción de capullos & $-0,750$ & $0,013 *$ & & \\
\hline & Desarrollo de juveniles & $-0,405$ & 0,245 & & \\
\hline \multirow[t]{3}{*}{ As soluble } & Cambio de peso & $-0,234$ & 0,515 & $-0,341$ & 0,335 \\
\hline & Producción de capullos & $-0,645$ & 0,061 & $-0,498$ & 0,172 \\
\hline & Desarrollo de juveniles & $-0,837$ & $0,005^{*}$ & $-0,794$ & $0,011^{*}$ \\
\hline
\end{tabular}

*Significativo con una probabilidad menor al $5 \%$. 
Se observó una notoria variación en el desarrollo de juveniles entre las estaciones, con menor producción en la estación CH2 y mayor en la estación SF1, con uno y 14 juveniles, respectivamente. Se observó un menor desarrollo de juveniles, significativamente diferente de las otras, en las estaciones $\mathrm{CH} 1$ y $\mathrm{CH} 2$ (Cuadro 3 y Figura 2). No se observó una correlación significativa entre el desarrollo de juveniles y las formas de cobre. Sin embargo, se evidenció una correlación significativa con las concentraciones de arsénico soluble, donde se destaca un bajo desarrollo de juveniles en concentraciones mayores que $21 \mu \mathrm{g} \mathrm{kg}^{-1}$ (Cuadro 4 y Figura 2).

Como señala Spurgeon et al. (1994), los parámetros reproductivos son más sensibles que la sobrevivencia

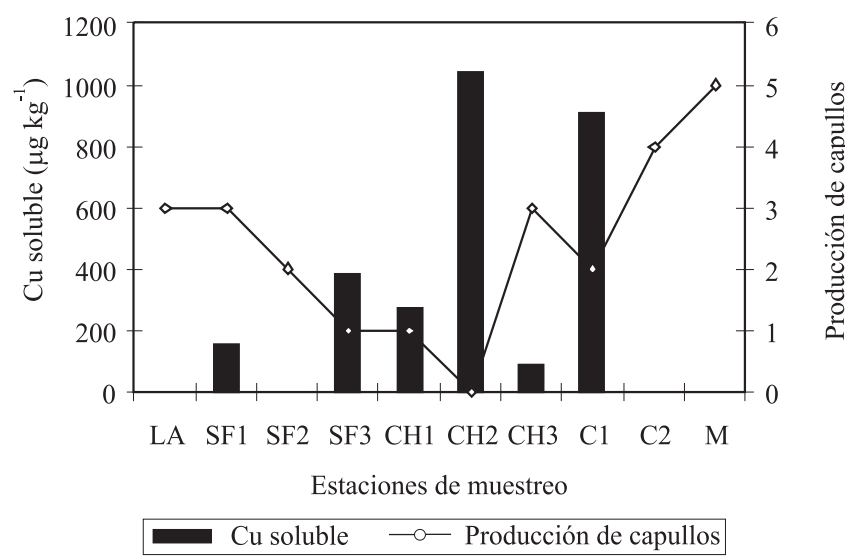

Figura 1. Relación entre las concentraciones de cobre soluble y la producción de capullos. (Estaciones de muestreo: LA: Los Andes; SF: San Felipe; CH: Chagres; C: Catemu; M: El Melón).

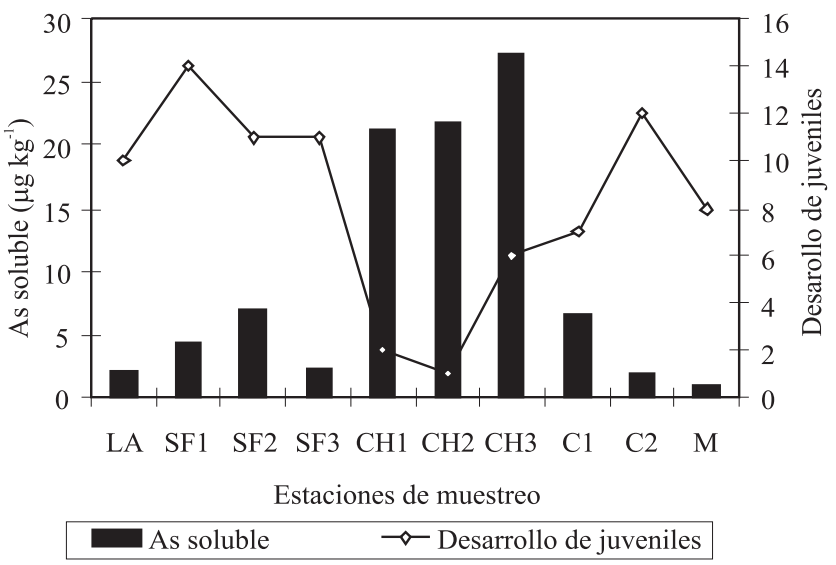

Figura 2. Relación entre las concentraciones de arsénico soluble y el desarrollo de juveniles. (Estaciones de muestreo: LA: Los Andes; SF: San Felipe; CH: Chagres; C: Catemu; M: El Melón). en los bioensayos de toxicidad crónica. Esto concuerda con los resultados del presente estudio. De acuerdo a nuestros resultados, se podría señalar que el cobre y arsénico actuarían en distintas etapas de desarrollo de la lombriz de tierra; ambos fueron tóxicos en la etapa de producción de capullos, mientras que el arsénico sólo en la etapa de desarrollo juvenil.

\section{Conclusiones}

1. Suelos agrícolas provenientes de áreas mineras, de la cuenca del rio Aconcagua, poseen potenciales características tóxicas para el desarrollo de la macrofauna edáfica.

2. El cobre y arsénico, en conjunto, disminuyeron la producción de capullos, mientras que sólo el arsénico disminuyó el desarrollo de juveniles.

\section{Agradecimientos}

Al FONDECYT (proyecto1050403) y a la Universidad de Valparaíso (proyecto DIPUV 06/2003), por el financiamiento de esta investigación; a Marcos Cisternas (Universidad Católica de Valparaíso), por sugerencias durante la realización de este trabajo.

\section{Referencias}

ADRIANO, D.C. Trace elements in terrestrial environments: biogeochemistry, bioavailability, and risks of metals. $2^{\text {nd }} e d$. New York: Springer, 2001. 867p.

AMONETTE, J. Methods for determination of mineralogy and environmental availability. In: DIXON, J.; SCHULZE, D. (Ed.). Soil mineralogy with environmental applications. Madison: Soil Science Society of America, 2002. p.153-197.

BADILLA-OHLBAUM, R.; GINOCCHIO, R.; RODRÍGUEZ, P.H.; CÉSPEDES, A.; GONZÁLEZ, S.; ALLEN, H.E.; LAGOS, G.E. Relationship between soil copper content and copper content of selected crop plants in Central Chile. Environmental Toxicology and Chemistry, v.20, p.2749-2757, 2001.

CORNELL, R.M.; SCHWERTMANN, U. The iron oxides: structure, properties, reactions, occurrences and uses. Weinheim: $\mathrm{VCH}$, 1996. 573 p.

DE GREGORI, I.; FUENTES, E.; ROJAS, M.; PINOCHET, H.; POTIN-GAUTIER, M. Monitoring of copper, arsenic and antimony levels in agricultural soils impacted and non-impacted by mining activities, from three regions in Chile. Journal of Environmental Monitoring, v.5, p.287-295, 2003.

DE GREGORI, I.; LOBOS, G.; LOBOS, S.; PINOCHET, H.; PONTINGAUTIER, M.; ASTRUC, M. Copper and selenium in rainwater, soils and alfalfa from agricultural ecosystems of Valparaiso region, Chile. Boletín de la Sociedad Chilena de Química, v.45, p.131-146, 2000. 
FISCHER, E.; KOSZORUS, L. Sublethal effects, accumulation capacities and elimination rates of $\mathrm{As}, \mathrm{Hg}$ and $\mathrm{Se}$ in the manure worm, Eisenia fetida (Oligochaeta, Lumbricidae). Pedobiologia, v.36, p.172178, 1992.

GINOCCHIO, R.; RODRÍGUEZ, P.H.; BADILLA-OHLBAUM, R.; ALLEN, H.E.; LAGOS, G.E. Effect of soil copper content and $\mathrm{pH}$ on copper uptake of selected vegetables grown under controlled conditions. Environmental Toxicology and Chemistry, v.21, p.1736-1744, 2002.

INTERNATIONAL ATOMIC ENERGY AGENCY (Viena, Austria). Quality aspects of research reactor operations for instrumental neutron activation analysis. Viena, 2001. 60p. (Technical document, 1218).

JANSSEN, R.; POSTHUMA, L.; BAERSELMAN, R.; DEN HOLLANDER, H.; VAN VEEN, R.; KABATA-PENDIAS, A. Agricultural problems related to extensive trace metal contents of soil. In: SALOMONS, W.; FORSTNER, U.; MADEN, P. (Ed.). Heavy metals, problems and solutions. Berlin: Springer-Verlag, 2005. p.3-18.

LANNO, R.; WELLS, J.; CONDER, J.; BRADHAM, K.; BASTA, $\mathrm{N}$. The bioavailability of chemicals in soil for earthworms. Ecotoxicology and Environmental Safety, v.57, p.39-47, 2003.

MARTÍNEZ, C.E.; McBRIDE, M.B. Dissolved and labile concentrations of $\mathrm{Cd}, \mathrm{Cu}, \mathrm{Pb}$, and $\mathrm{Zn}$ in aged ferrihydrite-organic matter systems. Environmental Science and Technology, v.33, p.745-750, 1999.

MARTÍNEZ, C.E.; McBRIDE, M.B. Solubility of $\mathrm{Cd}^{2+}, \mathrm{Cu}^{2+}, \mathrm{Pb}^{2+}$, and $\mathrm{Zn}^{2+}$ in aged coprecipitates with amorphous iron hydroxides. Environmental Science and Technology, v.32, p.743-748, 1998.

McBRIDE, M.B. Environmental chemistry of soils. New York: Oxford University Press, 1994. 406p.

McBRIDE, M.B.; SAUVÉ, S.; HENDERSHOT, W. Solubility control of $\mathrm{Cu}, \mathrm{Zn}, \mathrm{Cd}$ and $\mathrm{Pb}$ in contaminated soils. European Journal of Soil Science, v.48, p.337-346, 1997.

MAXWELL, J. Rock and mineral analysis. Oxford: Pergamon Press, 1968. 584p.

NATIONAL PETROGRAPHIC SERVICE. National Petrographic Service. Disponible en: <http://www.nationalpetrographic.com>. Leído el: 31 jan. 2007.

O'NEILL, P. Arsenic. In: ALLOWAY, B.J. (Ed.). Heavy metals in soils. London: Blackie Academic \& Professional, 1995. p.105-121.

ORGANISATION FOR ECONOMIC COOPERATION AND DEVELOPMENT (Paris, Francia). Draft guideline for the testing of chemicals: earthworm reproduction test (Eisenia foetidal andrei). Paris, 2000. 17p.

ORGANISATION FOR ECONOMIC COOPERATION AND DEVELOPMENT (Paris, Francia). Guidelines for the testing of chemicals: earthworm acute toxicity tests. Paris, 1984. 9p.

RÄTY, M.; HUHTA, V. Earthworm communities in birch stands with different origin in Central Finland. Pedobiologia, v.48, p.283291, 2004.

SADZAWKA, A.; CARRASCO, M.A.; GREZ, R.; MORA, M.L.; FLORES, H.; NEAMAN, A. Métodos de análisis de suelos recomendados para los suelos de Chile. Santiago: Instituto Nacional de Investigaciones Agropecuarias, 2006. 164p. (INIA. Serie actas, 34).

SAUVÉ, S.; McBRIDE, M.B.; HENDERSHOT, W.H. Ion-selective electrode measurements of copper (II) activity in contaminated soils. Archives of Environmental Contamination and Toxicology, v.29, p.373-379, 1995.

SAUVÉ, S.; McBRIDE, M.B.; NORVELL, W.A.; HENDERSHOT, W.H. Copper solubility and speciation of in situ contaminated soils: effects of copper level, $\mathrm{pH}$ and organic matter. Water, Air and Soil Pollution, v.100, p.133-149, 1997.

SCOTT-FORDSMAND, J.J.; WEEKS, J.M.; HOPKIN, S.P. Importance of contamination history for understanding toxicity of copper to earthworm Eisenia foetida (Oligochaeta: Annelida), using neutral-red retention assay. Environmental Toxicology and Chemistry, v.19, p.1774-1780, 2000.

SOIL SURVEY STAFF (Washington, Estados Unidos). Soil taxonomy: a basic system of soil classification for making and interpreting soil surveys. Washington: U.S. Dept. of Agriculture, Natural Resources Conservation Service, 1999. 869p.

SPARKS, D.L. Environmental soil chemistry. $2^{\text {nd }}$ ed. San Diego: Academic Press, 2003. 352p.

SPURGEON, D.J.; HOPKIN, S.P. Effects of metal-contaminated soils on the growth, sexual development, and early cocoon production of the earthworm Eisenia foetida, with particular reference to zinc. Ecotoxicology and Environmental Safety, v.35, p.86-95, 1996.

SPURGEON, D.J.; HOPKIN, S.P. Extrapolation of the laboratorybased OECD earthworm toxicity test to metal-contaminated field sites. Ecotoxicology, v.4, p.190-205, 1995.

SPURGEON, D.J.; HOPKIN, S.P.; JONES, D.T. Effects of cadmium, copper, lead and zinc on growth, reproduction and survival of the earthworm Eisenia foetida (Savigny): assessing the environmental impact of point-source metal contamination in terrestrial ecosystems. Environmental Pollution, v.84, p.123-130, 1994.

SPURGEON, D.J.; WEEKS, J.M.; VAN GESTEL, C.A.M. A summary of eleven years progress in earthworm ecotoxicology. Pedobiologia, v.47, p.558-606, 2003.

VAN DER WATT, H. Improved tables and simplified procedure for soil particle size analysis by the hydrometer method. South African Journal of Agricutural Sciences, v.9, p.911-916, 1966.

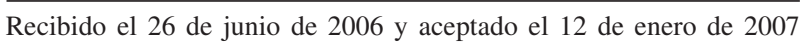

